\title{
A dual-band polarization insensitive metamaterial absorber with a single metal square patch for sensing application
}

Yongqiang Kang ( $\sim$ kyq_2000@sohu.com )

Shanxi Datong University https://orcid.org/0000-0001-6428-1807

Jun Wang

Xi'an Polytechnic University

Homgmei Liu

Shanxi Datong University - Yudong Campus: Shanxi Datong University

\section{Research Article}

Keywords: Dual-band, polarization insensitive, metamaterial, surface lattice resonance, sensing

Posted Date: February 22nd, 2021

DOI: https://doi.org/10.21203/rs.3.rs-149159/v2

License: (c) (i) This work is licensed under a Creative Commons Attribution 4.0 International License.

Read Full License 


\section{Abstract}

We proposed a dual-band polarization-insensitive metamaterial absorber consisting of merely the metal square patch and a continuous metal ground separated by a middle dielectric layer. Two resonance peaks derived from 'the fundamental resonance (with 97\% absorbance) and the surface lattice resonance (with 99\% absorbance) are realized. It is different from previous work the dual-band response is obtained by combining two resonances of different sizes. Moreover, a first-order diffraction mode of grating predicted the resonance wavelength of the proposed absorber. The surface electromagnetic field distributions of the unit-cell structure reveal the physical origin of the dual-band absorption. Importantly, the first absorption peak result from surface lattice resonance with narrow line-width has large sensitivity perform and high quality factor, which has significant potential in the application of biosensors and monitoring.

\section{Introduction}

Metamaterial absorbers with their unordinary properties have attracted attention of many scholars due to their promising applications such as sensor, cloaks, radars, and thermal emitters etc. [1-15]. These applications are mainly attributed to the unique properties of sub-wavelength metallic structure supporting localized surface plasmonic resonance modes [16-27]. Recently, it is shown the inherent losses of metallic structures can enhance absorption and achieve perfect absorption by specific structures [28-32]. Since then, metamaterial absorbers have attracted much attention and many of them have been proposed. Generally, metamaterial absorber can be classified as broadband and narrowband type based on their different application requirements. Broadband absorbers are extensively used in solar power harvesting and thermal emission [29,31]. While narrowband absorbers are applied for photodetectors and sensor $[9,14]$. Unfortunately, most of the metamaterial absorbers reported are single absorption bandwidth that are polarization-sensitive, which significantly obstacle their practical applications [20-23]. It is necessary to design dual- or multiband metamaterial absorbers with polarization-insensitive. The effective method is combining the resonance unit with different frequencies to obtain the dua-band or multiband response [33-38]. For example, Feng et al. demonstrated dual-band and multiband infrared absorber by horizontally arranging several subunits of different sizes into one unit cell [33]. Guo et al. presented ultra-broadband absorber by using four sub-wavelength resonators in the infrared region [34]. Zhang et al. reported a dual-band absorber by vertically stacking two distinct dielectrics [35]. However, the reported approaches face two challenging problems: large unit size problem and the difficulties of manufacturing technology at higher frequencies such as terahertz, infrared, and visible region.

In this paper, we demonstrate a polarization-insensitive dual-band metamaterial absorber constituted by merely a metal square patch, a dielectric space layer and a metal ground. It is found that there are two distinct absorption bands whose peak absorption about $97 \%$ in the fundamental mode and peak absorption about $99 \%$ with surface lattice resonance. The proposed absorber used the single pattered metallic structure by combining the fundamental resonance and surface lattice resonance, which is 
different from the results of previous report by overlapping or connecting various sized subunits to obtain dual-band response. Thus, it makes the proposed absorber much easier to construct than previously reported structures. Importantly, the absorption peak stem from surface lattice resonance has narrow linewidth and large sensitivity perform. These characteristic make it exhibit prominent sensing application. Furthermore, we demonstrate the proposed absorber can keep high absorption level at large angles of polarization.

\section{Structure And Design}

The proposed structure of the dual-band metamaterial absorber is shown in Fig. 1, which is consists of three layers. The entire structure is fabricated on a glass substrate. The top layer is a gold square patch. The middle layer is the dielectric layer, and the bottom layer is gold film. The thickness of gold square patch is $t=57 \mathrm{~nm}$, which have same side lengths $w=0.366$ um along the $\mathrm{x}$ and $\mathrm{y}$ axis. The middle dielectric layer had a dielectric constant of 1.96 [22] and a thickness $h=35 \mathrm{~nm}$. The thickness of the ground layer gold film is $L=0.1 \mathrm{um}$, and the lattice constant is $P=0.825 \mathrm{um}$. The dielectric constant of gold is from reference $[33,34]$. The finite difference time domain (FDTD) method is used to get the exact results. In the simulation process, the periodic boundary condition is applied along the $\mathrm{x}$ and $\mathrm{y}$ direction, and perfectly matched layers are set along the $z$ direction. The absorption of the structure can be obtained by $A=1-R-T$, where $R$ and $T$ is reflectivity and transmissivity, respectively. The transmissivity, while the thickness of the metal film $(L=0.1 \mathrm{um})$ is much larger than the skin depth. The perfect absorption can be achieved when the reflectivity $R$ approach to zero (i.e. the equivalent impedance of the proposed absorber structure matched to air).

\section{Results And Discussion}

\subsection{The absorption property under normal incidence}

Fig. 2 shows the reflectivity, transmittance and absorption of the dual-band metamaterial absorber. It is obvious that the reflectivity curve has two significant dips result from two distinct type resonances. The transmittance $T$ is zero owing to the presence of the metallic bottom layer, which blocks the transmission of the incident beam. Therefore, two discrete nearly perfect absorption peaks appear, the absorption of which is $99.2 \%$ (i.e., peak 1 ) and $97 \%$ (i.e., peak 2), respectively. The peak 1 at $723 \mathrm{~nm}$ has the absorption line-width of $16 \mathrm{~nm}$, which is about one-fifteenth of the peak at the $1254 \mathrm{~nm}$. The absorption line-width in this paper refers to the full width at half maximum (FWHM). Furthermore, the quality factor is defined as, where is the wavelength of the absorption peak [22,23]. The $Q$ value of peak 1 is about 46 , which is 7.2 times larger than that of peak 2. It is known that the narrow line-width and high $Q$ value are promised for sensing performance. This result is confirmed in below Fig. 7 and Fig. 8. It is demonstrated that the peak 1 has the sensitivity $550 \mathrm{~nm} / \mathrm{RIU}$ and figure of merit (FOM) 35, which are superior to better than that of the many sensing devices, see below Table 1 . 
The peak 1 with narrower absorption line-width and higher $Q$ come from the surface lattice resonance of the basic unit, while the peak 2 arises from the fundamental resonance. To give intuitional evidence, Fig. 3 shows absorption spectra with the different lattice constant $P$. It can be found that the peak 1 shift to the larger wavelength with the increase of the lattice constant $P$, while the shift of peak 2 is neglected. Therefore邓the physical mechanism of peak 1 is attributed to surface lattice resonance, which is distinct from the previously reported sandwich structure with only electromagnetic resonance or interference mechanisms $[24,25]$. The surface lattice resonance can be predicted approximately by the first-order diffraction mode of grating [23]. The resonance wavelength can by expressed as see formula 1 in the supplementary files.

Where $b$ is a numerical factor, and $\mathrm{n}$ is the refractive index of surrounding the device, and are the grating diffraction orders, and $P$ is the grating constant (or the unit period). The functional relationship of the resonance wavelength on the lattice period $P$ is shown in Fig. 4 . It can be seen that the resonance wavelength linearly rises with the lattice period $P$ increasing, which is same as shown in Fig. 3 . The theoretically predicted resonance frequency indicated by asterisk agrees roughly with the result of simulation when the lattice period $P$ varies from $0.765 \mathrm{um}$ to $0.825 \mathrm{um}$. The resonance wavelength of surface lattice resonance in simulation results has a little deviation for the theoretical value of the oneorder diffraction modes when the lattice period is smaller. This is because a surface lattice resonance involve the interplay of the dipole resonance and the first-order diffraction mode [22,23].

To reveal the absorption mechanisms of the two resonance peaks, Fig. 5 shows the electromagnetic field distribution of the proposed absorber. Figs. 5(a)-(c) are the amplitude of the electromagnetic field at the resonance wavelength $723 \mathrm{~nm}$. It is seen in Figs. 5(a)-(c) that the electromagnetic field distributions mainly concentrate symmetrically on the top surface edges of the metallic square patch and the dielectric layer, which indicate that the peak 1 is the combined effect of the localized resonance of metal square patch and the surface lattice resonance of the whole structure. Thus, the peak 1 is attributed to lattice resonance. Electromagnetic field distributions at the wavelength $1252 \mathrm{~nm}$ are presented in Figs. 5 (d)-(f). We found that the near field patterns are both symmetrically distributed on the edges of the metallic square patch, as shown in Figs. 5(d)-(f). It is demonstrated that the peak 2 results from the fundamental electric and magnetic dipole resonance. Therefore, we proposed a new approach to obtain dual-band absorber by combining two different resonance modes in a single pattern structure.

\subsection{The polarization property of the proposed absorber}

In many situations, it is desirable to design a polarization insensitive absorber [23, 24]. Figs. 6(a) and (b) show the dependence of the proposed dual-band absorber on the polarization angle for the TE and TM modes, respectively. It is found that the location of absorption peaks and absorbance remained unchanged as the polarization angle change from 0 to 90 degree at normal incidence. It is easily understood because of the high degree of symmetry for the metallic square patch in Fig 1.

\subsection{The sensing performance of the proposed absorber}


Since the first absorption peak has a high $Q$ value, it is promising for sensing application [22, 27]. For verification, Fig. 7 shows the dependence of the absorption spectrum when the surrounding refractive index (RI) from $n=1.0$ (air) to1.08 with an interval of 0.02. From Fig. 7, we can find that the peak 1 strongly depend on the RI (n), while the peak 2 has a weak effect. The functional relationship of the resonance peak 1 on the $\mathrm{RI}(\mathrm{n})$ is shown in Fig. 8 . It is shown that resonant peak redshift linearly with the increasing the RI of surrounding. Additionally, the absorption bandwidth and absorption are almost unchanged due to the impedance of the proposed absorber matched to the free space.

To evaluate the sensing performance of the proposed absorber, the sensitivity $(S)$ and the figure of merit $(F O M)$ are defined as follows [22,23]: see formula 2 in the supplementary files.

where and are the changes of the resonance wavelength and the RI, respectively. FWHM is the full-width at the half-maximum of the proposed device. From Eq. (2), the sensitivity and FOM of the peak 1 are 550 $\mathrm{nm} / \mathrm{RIU}$ and 35, respectively. This is larger than the value than previously reported light devices, see Table I [39-50].The high $Q$ and sensitivity have potential application in detection and sensing.

Table 1. Performance comparisons of the proposed device with the previous works.

\begin{tabular}{|lcc|}
\hline References & Q & FOM \\
39 & 11.6 & 2.3 \\
40 & 15 & 3 \\
41 & 8.5 & 4 \\
42 & 8.5 & 0.85 \\
43 & 7 & 0.5 \\
44 & 5.5 & 0.4 \\
45 & 40 & 1.5 \\
46 & 22.1 & 2.94 \\
47 & 7.8 & 0.7 \\
48 & 41 & 2.3 \\
49 & 58 & 7.5 \\
50 & 7 & 3.5 \\
\hline This paper & 46 & \\
\hline
\end{tabular}

\section{Conclusion}


A polarization-insensitive dual-band metamaterial absorber composed by only a metal square patch and a continuous metal ground separated by a middle dielectric layer is reported. Two distinct absorption peaks derived from 'the fundamental resonance and the surface lattice resonance are presented. Moreover, a first-order diffraction mode of the grating predicted the resonance wavelength of the proposed absorber. In addition, proposed dual-band absorber is insensitivity for all polarization angles of both TE and TM modes on normal incidence. Importantly, the absorption peak stem from surface lattice resonance has high $Q$ and a large sensitivity, which has significant potential in the application of biosensors.

\section{Declarations}

\section{Acknowledgements}

This research was financially supported by National Science Foundation for China (Grant NO. 11874245) and Shanxi Provincial Natural Science Foundation (Grant NO. 201801D121071, 201701D221096) and Scientific and Technological Innovation Project of Colleges in Shanxi Province (Grant NO. 2019L0741).

\section{Authors' contributions}

Y.Q. Kang and H.M. Liu conceived the concept. J. Wang performed simulation and wrote the manuscript. Y. Q. Kang analyzed the data. H.M. Liu guided the entire work. All authors read and approved the final manuscript.

\section{Availability of Data and Materials}

All data are fully available without restriction

\section{Competing Interests}

The authors declare that they have no competing interests.

\section{Author details}

${ }^{1}$ School of Physical Science and Electronics, Shanxi Datong University, Datong City 037009, People's Republic of China. ${ }^{2}$ School of Materials Science and Engineering, Xi'an Polytechnic University, Xi'an 710048, PR China. Innstitute of solid state physics, Shanxi Datong University, Datong, Shanxi 037009, China.

\section{Competing interests}

The authors declare that they have no competing interesting. 


\section{References}

[1]J. A. Schuller, E. S. Barnard, W. S. Cai, Y. C. Jun, J. S. White, and M. L. Brongersma, "Plasmonics for extreme light concentration and manipulation," Nat. Mater. 9, 193-204 , 2010.

[2]J. W. Mu, L. Chen, X. Li, W. P. Huang, L. C. Kimerling, and J. Michel, "Hybrid nano ridge plasmonic polaritons waveguides," Appl. Phys. Lett. 103, 131107, 2013.

[3] H. Lu, X. Liu, D. Mao, and G. Wang, "Plasmonic nanosensor based on Fano resonance in waveguidecoupled resonators," Opt. Lett. 37, 3780-3782, 2012.

[4]Y. K. Gong, X. Liu, K. Li, J. Huang, J. J. Martinez, D. R. Whippey, and N. Copner, "Coherent emission of light using stacked gratings," Phys. Rev. B 87, 205121, 2013.

[5] C. H"agglund and S. P. Apell, "Plasmonic near-field absorbers for ultrathin solar cells," J. Phys. Chem. Lett. 3, 1275-1285, 2012.

[6] J. W. Mu, X. Li, and W. P. Huang, "Compact Bragg grating with embedded metallic nano-structures," Opt. Express, 18, 15893-15900, 2010.

[7] J. Y. Ou, E. Plum, L. Jiang, and N. I. Zheludev, "Reconfigurable photonic metamaterials," Nano Lett. 11, 2142-2144, 2011.

[8]G. Wang, H. Lu, X. Liu, D. Mao, and L. Duan, "Tunable multi-channel wavelength demultiplexer based on MIM plasmonic nanodisk resonators at telecommunication regime," Opt. Express 19, 3513-3518 2011.

[9] I. M. Pryce, Y. A. Kelaita, K. Aydin, and H. A. Atwater, "Compliant metamaterials for resonantly enhanced infrared absorption spectroscopy and refractive index sensing," ACS Nano 5, 8167-8174, 2011.

[10] H. T. Chen, J. F. O’Hara, A. K. Azad, A. J. Taylor, R. D. Averitt, D. B. Shrekenhamer, and W. J. Padilla, "Experimental demonstration of frequency-agile terahertz metamaterials," Nature Photon. 2, 295-298, 2008.

[11]A. E. Cetin and H. Altug, "Fano resonant ring/disk plasmonic nanocavities on conducting substrates for advanced biosensing," ACSNano, 6, 9989-9995, 2012.

[12] J. Zi, Q. Xu, Q. Wang, C. Tian, Y. Li, X. Zhang, J. Han, and W. Zhang, "Antireflection-assisted alldielectric terahertz metamaterial polarization converter," Appl. Phys. Lett., 113, 101104, 2018.

[13] G. Kajtar, M. Kafesaki, E. N. Economou, and C. M. Soukoulis, "Theoretical model of homogeneous metal-insulator-metal perfect multi-band absorbers for the visible spectrum," J. Phys. D, 49, 055104, 2016.

[14] Dong. Wu, Ruifang,Li, Yumin, Liu, Zhongyuan Yu, Lei, Chen, Chang, Liu, Rui, Ma, and Han Ye, "Ultranarrow band perfect absorber and its application as plasmonic sensor in the visible region," Nanoscale Research Letters 12, 427, 2017. 
[15] Y. He, Q. Wu, and S. Yan, "Multi-band terahertz absorber at 0.1-1 THz frequency based on ultra-thin metamaterial," Plasmonics 14,1303-1310, 2019.

[16] J. Wu, F. Zhang, Q. Li, J. Chen, Q. Feng, and L. Wu, "Infrared fiveband polarization insensitive absorber with high absorptivity based on single complex resonator," Opt. Commun., 456, 124575, 2020.

[17] J. Zhang, J. Tian, L. Li, "A dual-band tunable metamaterial nearunity absorber composed of periodic cross and disk graphene arrays," IEEE Photon. J. 10, 4800512, 2018.

[18] J. Song, L. Wang, M. Li, and J. Dong, "A dual-band metamaterial absorber with adjacent absorption peaks," J. Phys. D, 51, 385105, 2018.

[19] Chunya, Luo, Dan, Li, Qin, Luo, Jie Yue, Peng,Gao, Jianquan yao, Furi Ling, “Design of a tunable multiband terahertz waves absorber," Journal of Alloys \& Compounds,652,18-24, 2015.

[20] Hongmei, Liu, Yongqiang, Kang. , Meng, T. , Tian, C. , \& Wei, G., "High photon absorptivity of quantum dot infrared photodetectors achieved by the surface plasmon effect of metal nanohole array,". Nanoscale Research Letters, 15(1), 2020

[21] J. Zhang, J. Tian, S. Xiao, L. Li, “Methodology for High Purity Broadband Near-Unity THz Linear Polarization Converter and its Switching Characteristics," IEEE Access, 8(1):46505-46517, 2020

[22] X.Y. Lu, R. Wan, and T. Zhang, "Metal-dielectric-metal based narrow band absorber for sensing applications," Optics Express 23, 29842, 2015.

[23] Ben Xin Wang, W. Q. Huang, and L. L. Wang . "Ultra-narrow terahertz perfect light absorber based on surface lattice resonance of a sandwich resonator for sensing applications." RSC Advances 7.68(2017):42956-42963.

[24] S. Ji, C. Jiang, J. Zhao, X. Zhang, \& Q. He, “Design of a polarization-insensitive triple-band metamaterial absorber," Optics Communications, 432, 65-70, 2019.

[25] X.P. Shen, Y. Yang, Y. Zang, J. Gu, J. Han, \& W. Zhang, et al. "Triple-band terahertz metamaterial absorber: design, experiment, and physical interpretation," Applied Physics Letters, 101(15), 207402-445, 2012.

[26] H. T. Chen, H. Yang, R. Singh, J. F. O'Hara, A. K. Azad, S. A. Trugman, Q. X. Jia, A. J. Taylor, "Tuning the resonance in high temperature superconducting terahertz metamaterials," Physical Review Letters, 105(24), 247402, 2010.

[27] I. M. Pryce, Y. A. Kelaita, K. Aydin, \& H. A. Atwater, “Compliant metamaterials for resonantly enhanced infrared absorption spectroscopy and refractive index sensing," ACS Nano, 5(10), 8167-8174, 2011. 
[28] J. Hao, L. Zhou, \&M. Qiu, "Nearly total absorption of light and heat generation by plasmonic metamaterials," Physical Review B Condensed Matter, 83(16), 5919-5926, 2011.

[29] H. Gao, C. Gu, Z. Zheng, Y. Chen, \& H. Y. Hao, "Nearly perfect absorption in a single-layer metallic grating with rectangular grooves on its front surface," Applied Physics B, 117(3), 875-883. 2014.

[30] P. V. Tuong, J. W. Park, V. D. Lam, W. H. Jang, S. A. Nikitov, \& Y. P. Lee, Dielectric and ohmic losses in perfectly absorbing metamaterials. Optics Communications, 295, 17-20. 2013.

[31] S.J. Li, P.X. Wu, H. Xiu, "Ultra-wideband and polarization-insensitive perfect absorber using multilayer metamaterials, lumped resistors, and strong coupling effects," Nanoscale Research Letters. 2018.

[32] N. I. Landy, S. Sajuyigbe, J. J. Mock, D. R. Smith, \& W. J. Padilla,. "A perfect metamaterial absorber". Physical Review Letters, 100(20), 207402, 2008.

[33] R. Feng, J. Qiu, L.H., Liu., "Parallel LC circuit model for multi-band absorption and preliminary design of radiative cooling," Optics Express.22,S7, A1713-1724, 2014.

[34] W.L. Guo, Y. Liu, and T. Han, "Ultra-broadband infrared metasurface absorber," Optics Express, 24.18, 20586-20592, 2016.

[35] N. Zhang, P. Zhou, D. Cheng, X. Weng, \& L. Deng, "Dual-band absorption of mid-infrared metamaterial absorber based on distinct dielectric spacing layers," Optics Letters, 38(7), 1125-7, 2013.

[36] D. Cheng, J. Xie, H. Zhang, C. Wang, \& L. Deng, "Pantoscopic and polarization-insensitive perfect absorbers in the middle infrared spectrum," JOSA. B, 29(6), 1503-1510, 2012.

[37] Jiayun Wang, Rongcao Yang,jinping Tian, Chenxin Wei, Wenmei Zhang, "A Dual band absorber with wide-angle and polarization-insensitivity," IEEE Antennas and wireless Propagation letters. 05,18,2018.

[38] G. Yoon, S. So, M.Y. Kim, J.H. Mun, R.M. Ma, J. S. Rho, “Electrically tunable metasurface perfect absorber for infrared frequencies," Nano Covergence, 4:36, 2017.

[39] L. Cong, S. Tan, R. Yahiaoui, F. Yan, W. Zhang, and R. Singh, "Experimental demonstration of ultrasensitive sensing with terahertz metamaerial absorbers: A comparison with the metasurfaces," Appl. Phys. Lett., 106,031107, 2015.

[40] R. Yahiaoui, S. Tan, L. Cong, R. Singh, F. Yan, and W. Zhang, "Multipectra terahertz sensing with highly flexible ultrathin metamaterial absorber," J. appl. Phys., 118, 083103, 2015.

[41] X.Hu, G. Xu, L. Wen, H. Wang, Y. Zhao, Y. Zhang, D.R.S. Cumming and Q. Chen, “Metamaterial absorber integrated microfluidic terahertz sensors," Laser Photonics Rev., 10. 962. 2016,

[42] Y. Li, X. Chen, F. Hu, D. Li, H. Teng, Q. Rong, W. Zhang, J. Han, and H. Liang, “Four resonators based high sensitive terahertz metamaterial biosensor used for measuring concentration of protein," J. Phys. D, 
52, 095105, 2019.

[43] M. Islam, S. J. M. Rao, G. Kumar, B. P. Pal, D. R. Chowdhury, "Role of resonance modes on terahertz metamaterials based thin film sensors," Sci. Rep., 7, 7355, 2017.

[44] F. Shen, J. Qin, and Z. Han, "Planar antenna array as a highly sensitive terahertz sensor," Appl. Opt., 58, 540, 2019.

[45] I. Al-Naib, "Thin-film sensing via fano resonance excitation in symmetric terahertz metamaterials," Journal of Infrared, Millimeter, and Terahertz Waves, 39, 1, 2018.

[46] A. S. Saadeldin, M. F. O. Hameed, E. M. A. Elkaramany, and S. S. A. Obayya, "Highly sensitive terahertz metamaterial sensor," IEEE SensorsJ., 19, 7993, 2019.

[47] K. Meng, S. J. Park, A. D. Nurnett, T. Gill, C. D. Wood, M. Rosamond, L. H. Li, L. Chen, D. R. Bacon, J. R. Freeman, P. Dean, Y. H. Ahn, E. H. Linfield, A. G. Davies, and J. E. Cunningham, "Increasing the sensitivity of terahertz split ring resonator metamaterials for dielectric sensing by localized substrate etching," Opt. Express, 27, 23164, 2019.

[48] I. Al-Naib, "Biomedical Sensing with conductively coupled terahertz metamaterial resonators," IEEE J. Select. Top. Quantum Electron., 23, 4700405, 2017.

[49] Q. Xie, G.X. Dong, B.X. Wang,and W.Q. Huang, "High-Q fano resonance in terahertz frequency based on an asymmetric metamaterial resonator," Nanoscale Res. 13囚294, 2018.

[50] R. Yahiaoui, A.C. Strikwerda, and P.U. Jepsen, "Terahertz palsmonic structure with enhanced sensing capabilities," IEEE Sensors, 16, 24842016.

\section{Figures}




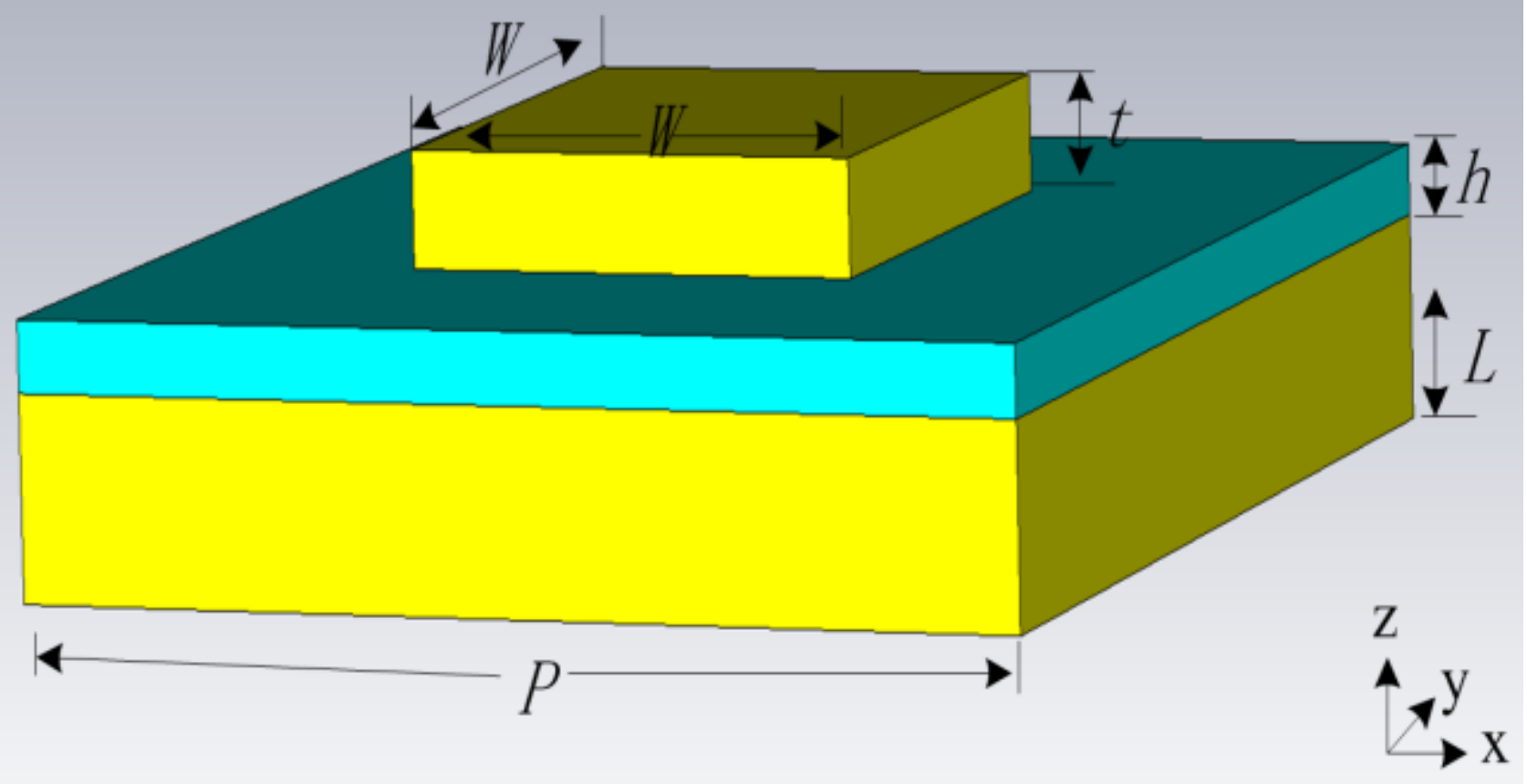

\section{Figure 1}

(color online) Schematic of the proposed dual-band absorber. The yellow regions are gold, and light blue region is dielectric. $w$ and $t$ represent respectively, the side lengths of the rectangular gold patch and its height . $\mathrm{h}$ and $\mathrm{L}$ denote the thickness of the dielectric layer and the gold film. $\mathrm{P}$ is the lattice constant

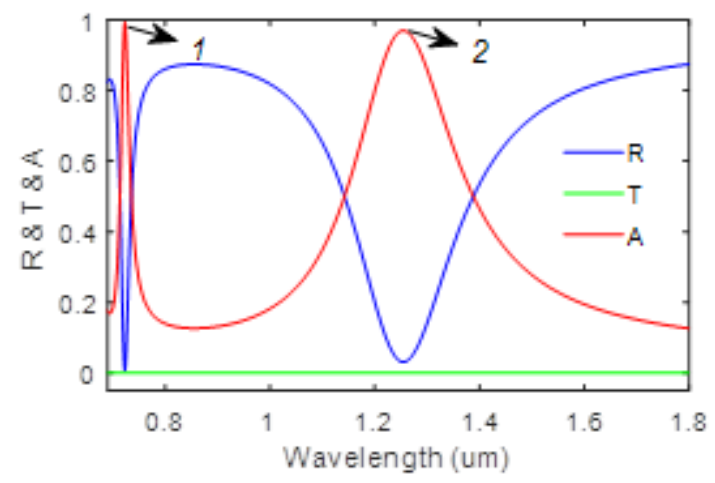

Figure 2

(color online) Refection, transmission and absorption spectra of the dual-band absorber. Parameters: $P=0.825 u m, w=0.366$ um, $t=0.057 u m, h=35 n m, L=0.1 u m$. 


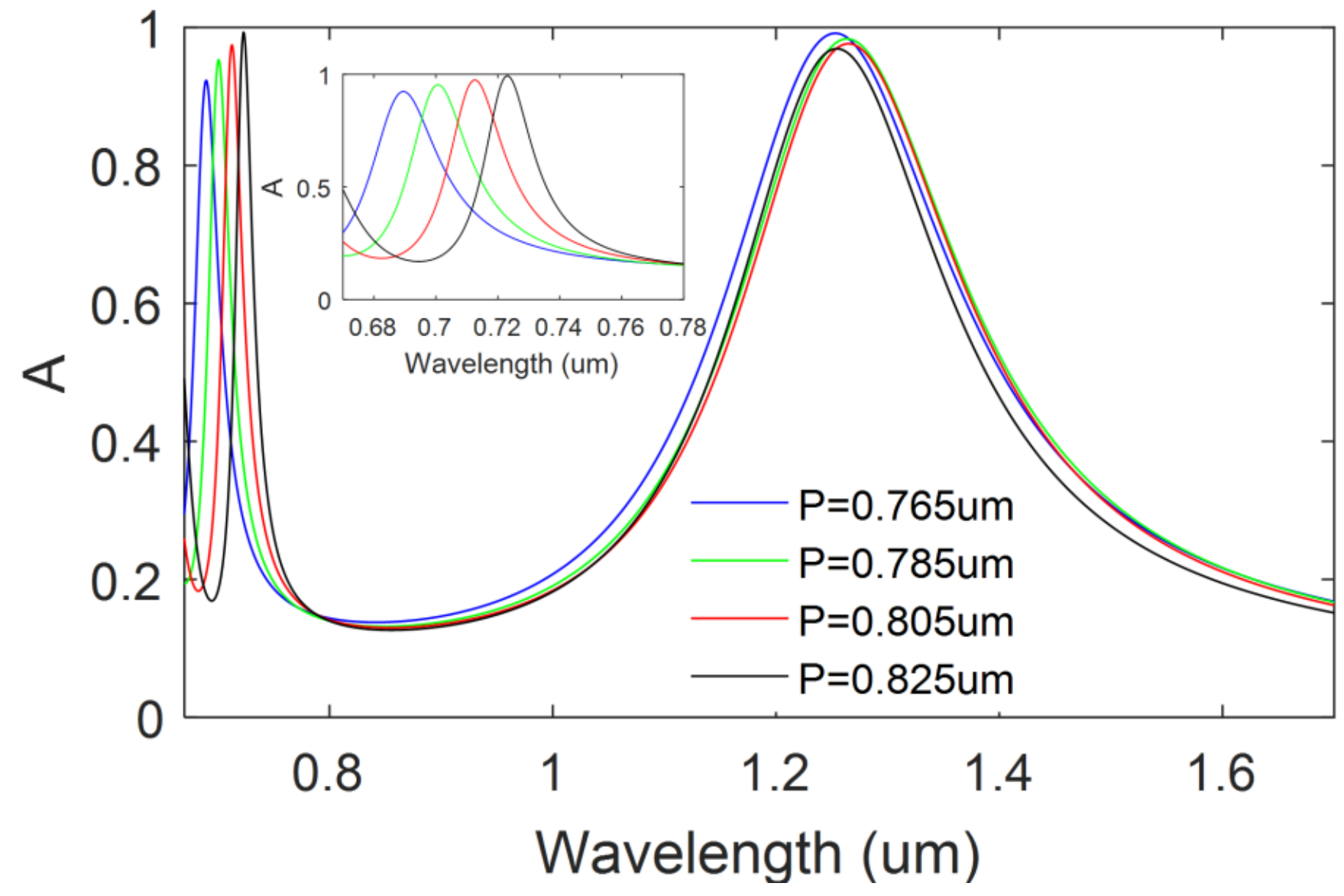

Figure 3

(color online) Dependence of absorption spectrum on the changes of lattice period $\mathrm{P}$ of the dual-band absorber. The inset figure shows the dependence of absorption spectrum of the resonance mode 1. Parameters: $P=0.825 u m, w=0.366 u m, t=0.057 u m, h=35 n m, L=0.1 u m$.

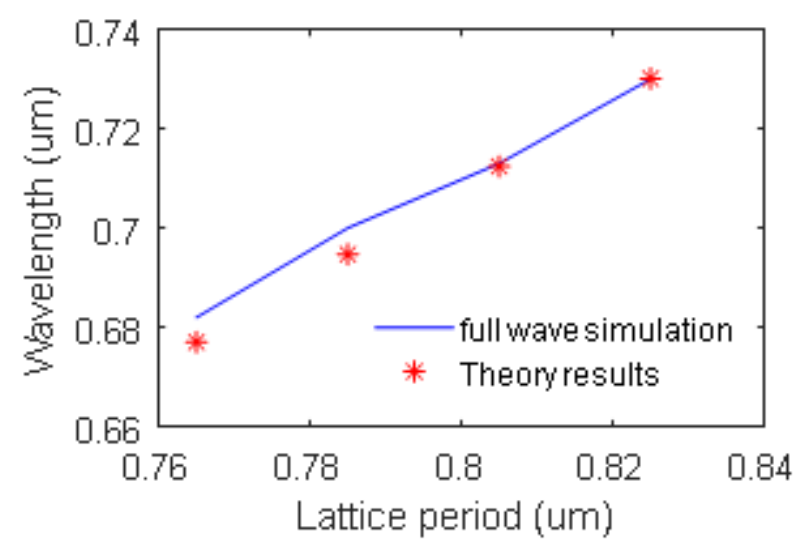

Figure 4

(color online) Comparison between the predicted resonance wavelength and results obtained from the full-wave simulation for different lattice period P. Parameters: $P=0.825 u m, w=0.366 u m, t=0.057 u m$, $\mathrm{h}=35 \mathrm{~nm}, \mathrm{~L}=0.1 \mathrm{um}$. 

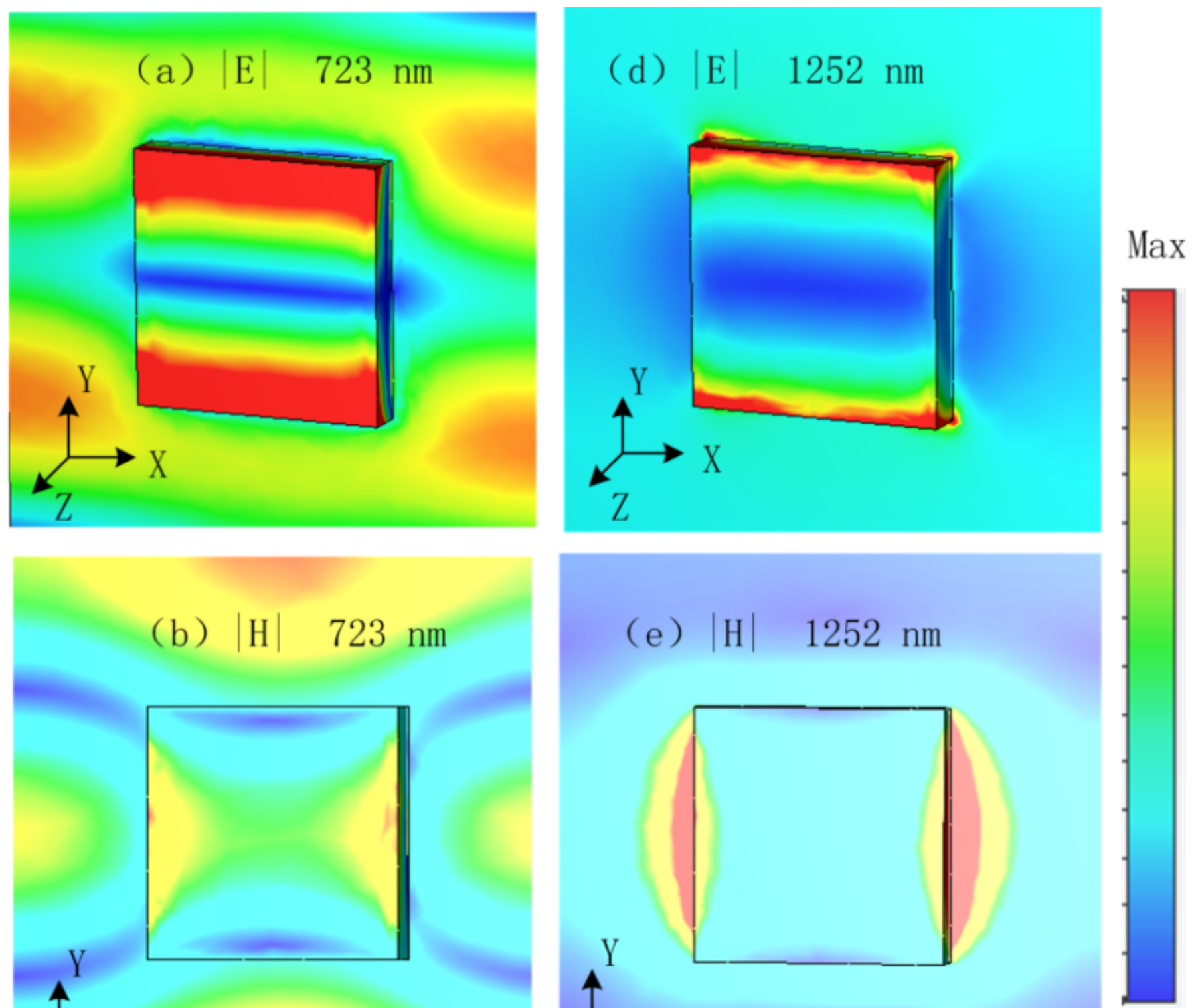

(b) $|\mathrm{H}| \quad 723 \mathrm{~nm}$

\section{(e) $|\mathrm{H}| 1252 \mathrm{~nm}$}
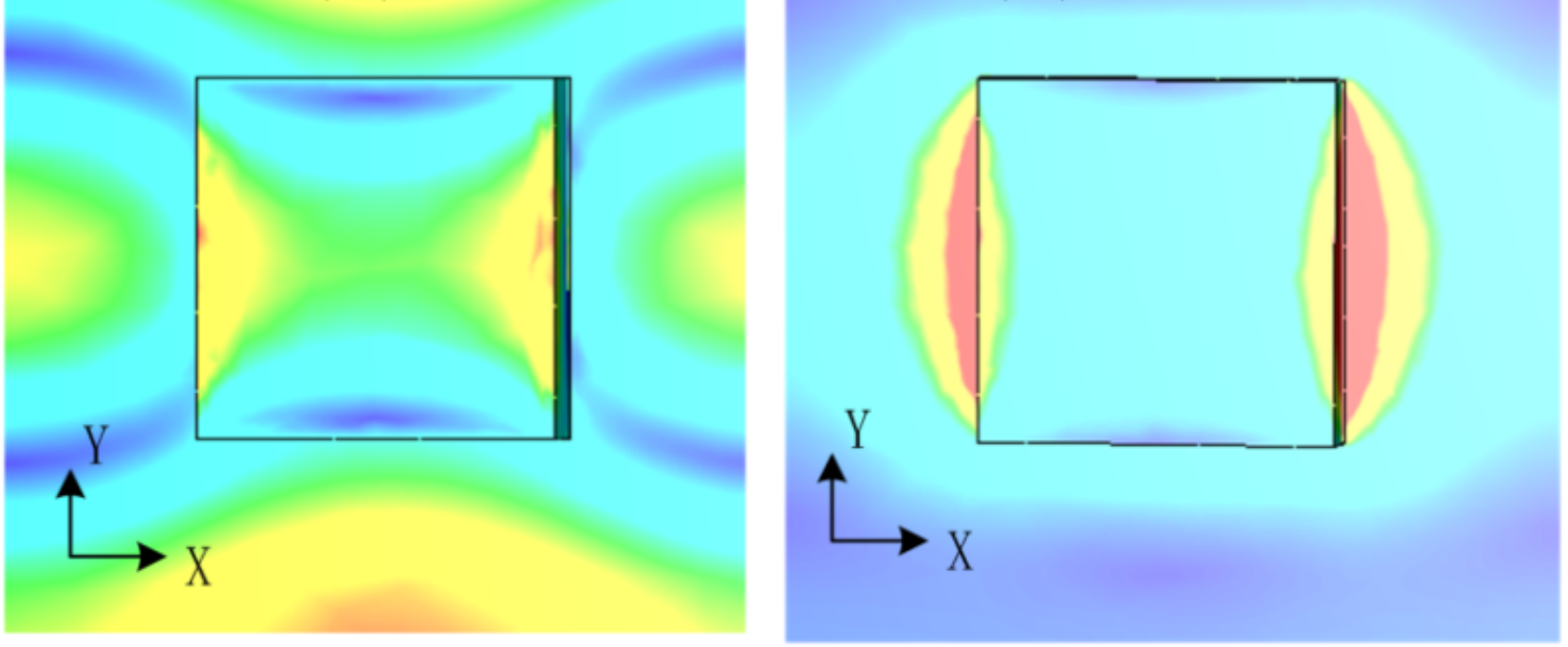

Min

\section{(c) $|\mathrm{H}| \quad 723 \mathrm{~nm}$}

\section{(f) $|\mathrm{H}| 1252 \mathrm{~nm}$}
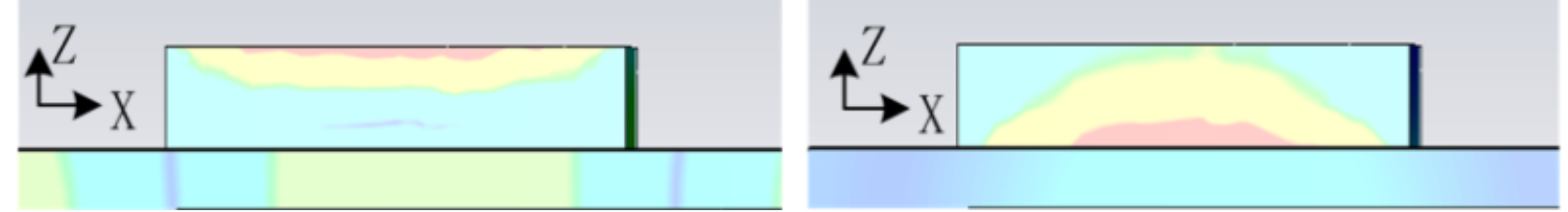

Figure 5

(color online) (a),(b)and (c) electromagnetic field distribution at resonance wavelength $723 \mathrm{~nm}$; (d), (e) and (f) electromagnetic field distribution at resonance wavelength $1252 \mathrm{~nm}$. Parameters: $P=0.825 \mathrm{um}$, $w=0.366$ um, $t=0.057 u m, h=35 \mathrm{~nm}, \mathrm{~L}=0.1 \mathrm{um}$. 

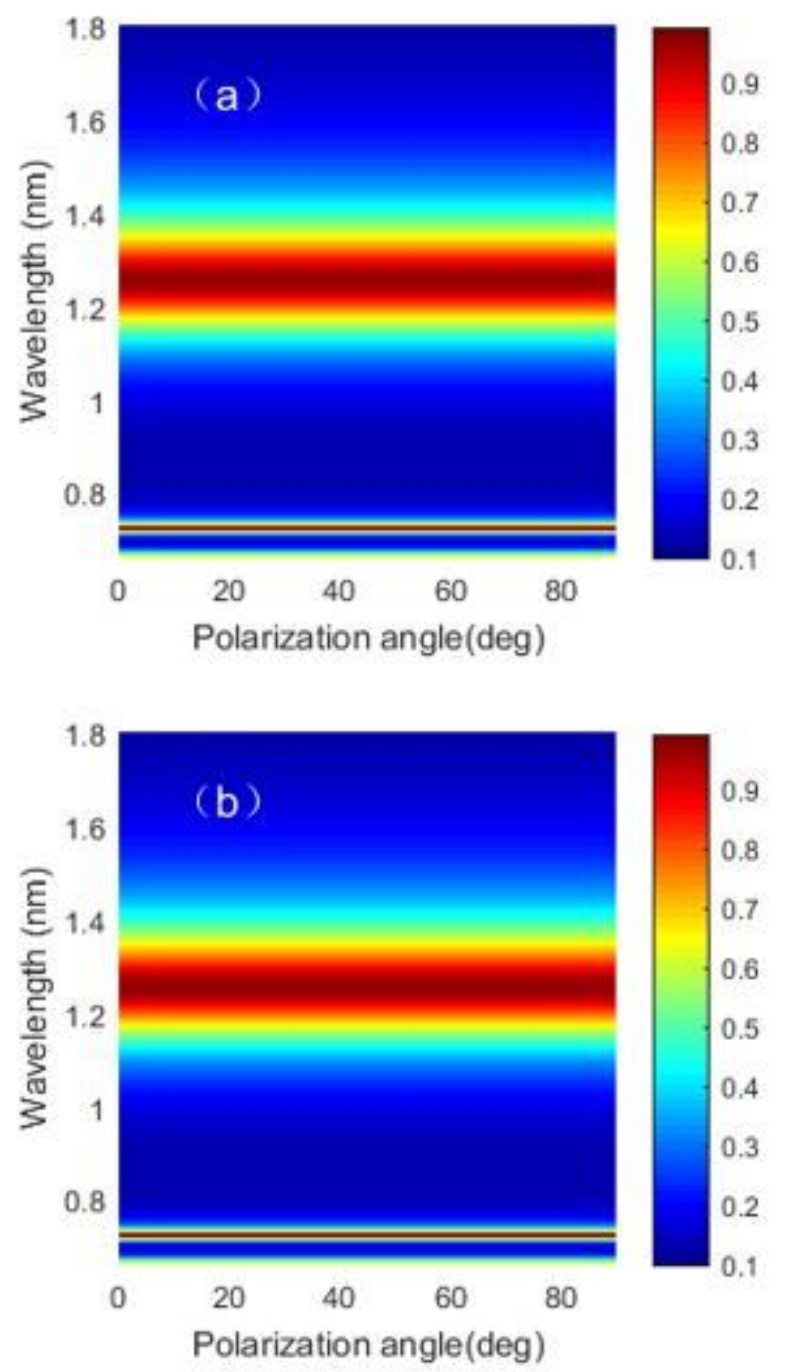

Figure 6

(color online) Simulated absorbance for different polarization angle: (a) TE, (b) TM. Parameters: $P=0.825 u m, w=0.366$ um, $t=0.057 u m, h=35 n m, L=0.1 u m$. 


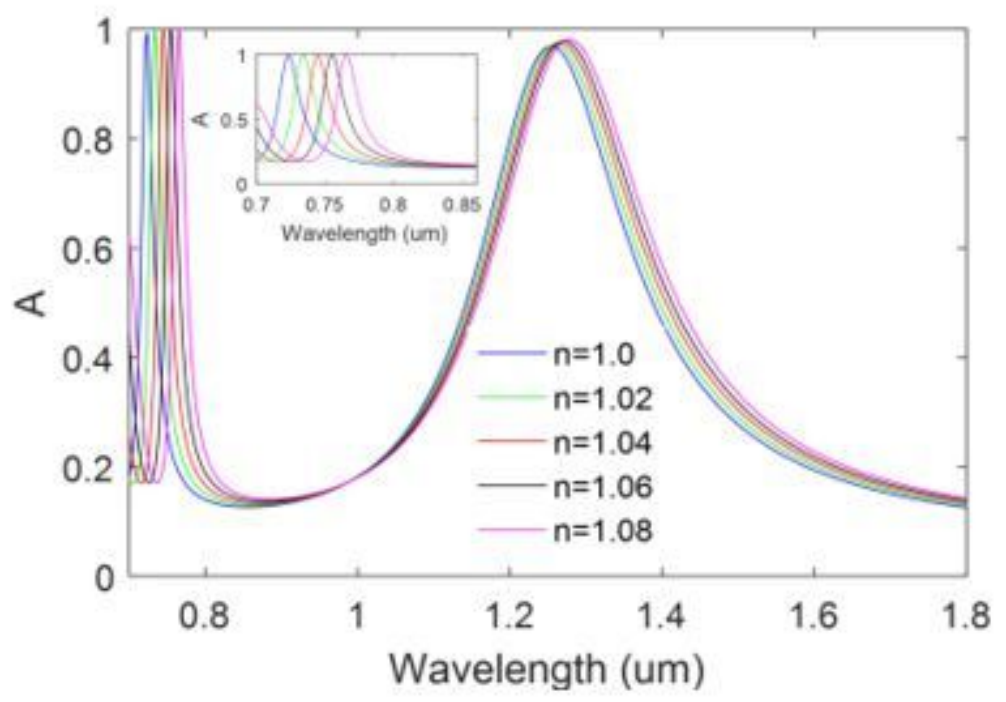

Figure 7

(color online) Dependence of the absorption spectrum on the changes of refractive index (n) of the surrounding; The inset figure shows the dependence of absorption spectrum of the resonance mode 1. Parameters: $P=0.825 u m, w=0.366$ um, $t=0.057 u m, h=35 n m, L=0.1 u m$.

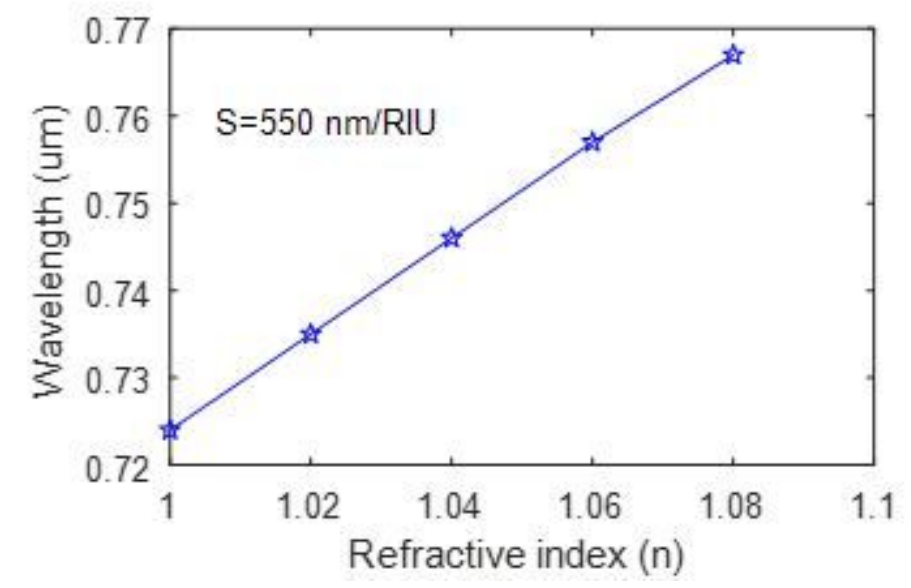

Figure 8

(color online) Relationship between the resonance wavelength and refractive index. Parameters: $P=0.825 u m, w=0.366 u m, t=0.057 u m, h=35 n m, L=0.1 u m$.

\section{Supplementary Files}

This is a list of supplementary files associated with this preprint. Click to download.

- formulas.docx 